\title{
Densidad de la madera de 59 especies del orden Sapindales procedentes de bosques naturales brasileños
}

\author{
Wood density of 59 tree species in the order Sapindales from natural \\ forests in Brazil
}

\author{
Guillermo Riesco Muñoz ${ }^{*}$, José Imaña Encinas' y José Elías de Paula²
}

\begin{abstract}
Universidad de Santiago de Compostela. Departamento de Ingeniería Agroforestal. Unidad de

2 Universidade de Brasilia. Departamento de Botánica. * Autor de correspondencia. (guillermo.riesco@usc.es Gestión Forestal Sostenible. Lugo, España
\end{abstract}

\section{RESUMEN}

Para estimar la biomasa leñosa a partir de las existencias maderables en volumen obtenidas en inventarios forestales, se determinó la densidad básica de la madera en 59 especies arbóreas, para algunas de las cuales escasea la información publicada sobre las características de su madera. Se trata de especies pertenecientes a las familias Anacardiaceae, Burseraceae, Meliaceae, Rutaceae, Sapindaceae y Simaroubaceae, todas ellas incluidas en el orden Sapindales. La densidad básica se obtuvo a partir de muestras procedentes de inventarios forestales realizados en las dos últimas décadas en diversas regiones del Brasil. Se comprobó que la mayor parte de las especies analizadas (68\%) presentan maderas livianas o semipesadas (densidad básica entre 0,51 y 0,80) aunque la densidad se mostró muy variable entre especies, desde 0,38 para Simarouba amara hasta 1,25 para Scbinopsis lorentziii. Se encontró información bibliográfica sobre densidad básica de la madera para tres cuartas partes de las especies objeto de estudio, resultando que según referencias bibliográficas la madera analizada en este trabajo es $21 \%$ más densa que la procedente de las mismas especies en otras regiones. Este trabajo aporta información sobre especies de maderas poco conocidas hasta el momento. Así mismo, las especies arbóreas del orden Sapindales procedentes de Brasil y que fueron analizadas en este trabajo, destacan por la alta densidad básica de su madera, por lo que contribuyen de forma especialmente eficaz a la acumulación de biomasa y a la correspondiente fijación de carbono.

PALABRAS CLAVE: bosque tropical, densidad básica, fijación de carbono, inventario forestal, tecnología de la madera.

\section{ABSTRACT}

The basic density of wood from 59 tree species was determined in order to estimate the weight of woody biomass from the volume of wood measured in forest inventories. Published information about wood properties of some of these species is scarce. The 59 species considered belong to the families Anacardiaceae, Burseraceae, Meliaceae, Rutaceae, Sapindaceae and Simaroubaceae, all of which are included in the order Sapindales. The basic density was determined in samples obtained in forest inventories performed in the last two decades in various regions of Brazil. Most of the species analyzed (68\%) had light or semi-heavy wood (basic density between 0,51 and 0,80), although the density varied widely between species, ranging from 0,38 for Simarouba amara to 1,25 for Schinopsis lorentziii. A literature search revealed data on the basic density of the wood of three quarters of the species under study. According to this data, the wood considered in the present study is $21 \%$ more dense than wood from the same species growing in other regions. This study provides new information on wood of tree species about which very little was previously known. The analyzed Brazilian tree species in the order Sapindales were found to be important because of the high basic density of their wood. These trees thus contribute efficiently to biomass accumulation and to the corresponding carbon fixation.

KEYWORDS: tropical forest, basic density, carbon fixation, forest inventory, wood technology. 


\section{INTRODUCCIÓN}

La cubierta forestal de Brasil es de 463 millones de hectáreas y $98 \%$ de esta cobertura son bosques naturales, localizados principalmente en el norte del país. Esta vasta extensión incluye los 356 millones de hectáreas que forman el bioma amazónico, que constituye la mayor cubierta arbórea tropical de la Tierra, la Caatinga, con 49 millones de hectáreas, y los 11 millones de hectáreas que forman el Pantanal, que es la mayor llanura inundable del mundo. Sin embargo, solo 19\% del volumen de las cortas de madera del país procede de masas forestales naturales (Eckardt, Rodrigues Nascimento, Nazário de Alameida y Vieira de Abreu, 2015). Se pretende que para 2030 haya 30 millones de hectáreas de bosque tropical brasileño sometido a planes de gestión forestal racionales, lo que supone multiplicar por diez la superficie de bosque tropical que es gestionada actualmente de forma sostenida. Estos planes tendrán entre sus objetivos el mantenimiento de carbono en forma de biomasa, reduciendo así la presencia de carbono en la atmósfera y mitigando de forma indirecta la contribución de Brasil al cambio climático por exceso de concentración de carbono en la atmósfera. La superficie que sería puesta en producción corresponde aproximadamente a 7\% de la extensión del bosque amazónico y permitiría atender a todo el consumo nacional de maderas tropicales. La propuesta para 2030 también incluye la erradicación, en lo posible, de las cortas ilegales de madera, con autorizaciones de corta basadas en inventarios forestales que sean de conocimiento público y en los que se informe de especies y existencias maderables en volumen. Un objetivo adicional es el estudio de la aptitud tecnológica de las especies forestales nativas menos conocidas (Rech, 2015).

A partir de la información pública sobre existencias maderables en volumen, procedente de inventarios forestales, es posible obtener las toneladas de biomasa en pie y de carbono fijado correspondientes si se dispone del valor medio representativo de la densidad básica para cada especie arbórea inventariada. Por ello, es necesario conocer la densidad básica de las especies arbóreas para efectuar las transformaciones precisas de existencias en volumen de madera a su correspondiente biomasa y fijación de carbono.

En el norte y oeste del Brasil se localizan los bosques tropicales de frondosas de hoja perenne (bosques siempre verdes), en zonas de temperaturas elevadas y precipitaciones repartidas a lo largo de todo el año, sin una estación seca marcada. Es característica de estas formaciones vegetales la alta densidad del arbolado (número de pies por hectárea), el gran volumen de existencias de madera y la multiplicidad de doseles de copas (dosel de árboles gigantes, a unos $50 \mathrm{~m}$ de altura, dosel de árboles menores, que alcanzan los $30 \mathrm{~m}$, y doseles inferiores para especies de sombra). Es el típico bosque pluriespecífico en el que se encuentra la caoba, también conocida por mabogany wood (Swietenia macrophylla King), la madera más apreciada del mundo junto con la teca (Tectona grandis L. f.), y otras maderas preciosas para ebanistería (Peraza Oramas y González Álvarez, 1973).

Gran parte de la biomasa vegetal del ecosistema en la región amazónica se acumula en las especies forestales arbóreas y la mayoría de la biomasa del estrato arbóreo corresponde a la fracción leñosa (fustes, ramas y ramillas). Sin embargo, se dispone de escasa información publicada acerca de la contribución de los órganos leñosos de dichas especies a las existencias de biomasa en términos de peso. En consecuencia, no está cuantificada suficientemente la cantidad de carbono atmosférico que fijan determinadas especies en sus tejidos leñosos, sobre todo aquellas especies menos conocidas y otras más conocidas, pero de procedencias poco exploradas (Brown, 1997). Para evaluar el papel de estas especies como organismos fijadores de carbono se necesita conocer detalladamente su madera, por ser la fracción leñosa la más abundante en la biomasa arbórea.

La densidad es una de las propiedades físicas de la madera que más aportan al conocimiento de este material (p.e. Mettem y Richens, 1991; Williamson y Wiemann, 2010) y entre las diferentes variables que cuantifican la densidad de la madera se encuentra la densidad básica, que se obtiene por cociente entre la masa anhidra de una pieza 
y el volumen de la misma a saturación de humedad. Es una propiedad física habitualmente empleada como indicador de calidad de la madera (p.e. Desch y Dinwoodie, 1996; Forest Products Laboratory, 2010; Zobel y Buijtenen, 1989) pero también es un coeficiente que permite efectuar la transformación de volumen de biomasa leñosa en verde a cantidad de materia seca, punto de partida para efectuar estimaciones de carbono en la fracción leñosa del arbolado en pie. Además, la densidad de la madera influye en la conductancia hidráulica (Li et al., 2016; Savi et al., 2017) y en las estrategias ecológicas de las plantas leñosas para controlar la proporción de carbono invertido por unidad de volumen de fuste (Martínez-Cabrera, Jones, Espino y Jochen Schenk, 2009).

En el estudio de la densidad de la madera debe tenerse en cuenta que es una variable generalmente sujeta a una significativa variación entre individuos (Williamson y Wiemann, 2010; Zobel y Buijtenen, 1989) y que, además, la densidad de la madera no es homogénea dentro de un mismo árbol, dado que depende fuertemente de la edad cambial, lo cual se traduce en una significativa variación de la densidad en la dirección radial del fuste (de la médula hacia la corteza) y en la dirección axial del mismo (de la base a la parte superior del fuste) (Desch y Dinwoodie, 1996; Kollmann, 1959). Por ello, en los trabajos de campo, la toma de muestras de madera para ensayos debe efectuarse a diferentes alturas del fuste, para captar la variabilidad a lo largo del mismo, o bien se debe escoger siempre el material a la misma altura, para eliminar del análisis estadístico la fuente de variación que constituye la altura en el fuste. Así mismo, el material de ensayo debe tomarse a diferentes profundidades del tronco, para abarcar de este modo la variación de la densidad de la madera en dirección radial (Williamson y Wiemann, 2010).

Entre las especies forestales nativas de Brasil menos conocidas por las características de su madera se encuentran algunas pertenecientes al orden Sapindales (familias Anacardiaceae, Burseraceae, Meliaceae, Rutaceae, Sapindaceae y Simaroubaceae). La familia Anacardiaceae comprende unos 70 75 géneros (49 de ellos monotípicos) y en torno a 600 especies, con maderas de colores diversos (amarillas, rojas, verdes, granates, jaspeadas) y densidad muy variable. Entre las especies más importantes desde el punto de vista maderero se encuentran el espave (Anacardium excelsum), el Gonzalo Alves (Astronium fraxinifolium y Astronium graveolens) y el quebracho (Schinopsis lorentrii). Entre las especies de la familia también se encuentra el mango (Mangifera indica L.), que es una especie exótica en Brasil (Devesa Alcaraz y Carrión García, 2012; Harrington, 2008; Izco, 2009; Peraza Oramas y González Álvarez, 1973; Strasburger y Sitte, 2004; Zomlefer, 2004).

La familia Burseraceae incluye 16-18 géneros y casi 600 especies, que son árboles y matorrales del Trópico. Muchas especies arbóreas tienen interés comercial por sus maderas, que con frecuencia son brillantes, algo aromáticas y de densidad muy diversa. Entre las especies más importantes desde el punto de vista maderero se encuentran el almácigo (Bursera simaruba) y el azucarero (Tetragastris balsamifera) (Devesa Alcaraz y Carrión García, 2012; Izco, 2009; Peraza Oramas y González Álvarez, 1973; Strasburger y Sitte, 2004).

La familia Meliaceae comprende 52 géneros y 621 especies de árboles perennifolios (Devesa Alcaraz y Carrión García, 2012). A esta familia pertenecen las maderas para ebanistería más importantes del mundo (Tomazello Filho, Botosso y Lisi, 2000). Las más destacadas por su interés maderero (caobas, caobillas, cedros) son maderas rojizas, brillantes, a veces olorosas, y de densidad media. Son importantes la cancharana (Cabralea canjerana), la andiroba (Carapa guianensis), el cedro de Sudamérica (Cedrela sp.), la guarea (Guarea sp.) y la caoba (Swietenia macrophylla) (Miller, 1990; Peraza Oramas y González Álvarez, 1973). Entre las especies de esta familia también se encuentra el cinamomo o agriaz (Melia azedarach), que es originario del noroeste de la India y de Irán pero se encuentra asilvestrado en Europa desde la Edad Media y también se encuentra presente en Brasil. Es especie ornamental y su madera se usa para viguerío (Izco, 2009).

La familia Rutaceae comprende cerca de 161 géneros y numerosas especies con importancia económica, entre las 
que se encuentran las especies productoras de la madera de satén, que destacan por su brillo. Las maderas de esta familia son de densidad media o elevada y muchas tienen un aroma característico, como el sándalo venezolano (Amyris balsamifera) o las especies del género Zanthoxylum. Una especie importante desde el punto de vista maderero es el boj brasileño (Euxylophora paraensis) (Devesa Alcaraz y Carrión García, 2012; Izco, 2009; Peraza Oramas y González Álvarez, 1973).

La familia Sapindaceae comprende entre 135 y 147 géneros y unas 2215 especies de árboles, arbustos y bejucos (Devesa Alcaraz y Carrión García, 2012; Izco, 2009; Strasburger y Sitte, 2004; Zomlefer, 2004). Es característico en los árboles de la familia el duramen amarillo, rosado o pardo rojizo, no distinguible de la albura. Su densidad puede ser de media a muy elevada (Peraza Oramas y González Álvarez, 1973). Entre las especies de esta familia se encuentra el palo de jabón (Sapindus saponaria).

La familia Simaroubaceae comprende unos 20-30 géneros y 100-150 especies. Presenta maderas muy variadas, generalmente de colores claros y muy brillantes. Es una madera importante desde el punto de vista industrial la simaruba o aceituno (Simaruba amara y Simaruba glauca) (Devesa Alcaraz y Carrión García, 2012; Izco, 2009; Peraza Oramas y González Álvarez, 1973; Strasburger y Sitte, 2004).

\section{OBJETIVOS}

Se pretende determinar la densidad básica de la madera de 59 especies forestales del Orden Sapindales (familias Anacardiaceae, Burseraceae, Meliaceae, Rutaceae, Sapindaceae y Simaroubaceae) procedentes de los biomas Sabanas Brasileñas, Pantanal, Caatinga y Amazonas (Brasil), comparando los resultados con los obtenidos en bibliografía.

\section{MATERIALES Y MÉTODOS}

A partir del año 2000, en todos los inventarios forestales realizados por los autores del presente trabajo, se siguió un proceso metodológico riguroso y normalizado para la recogida de muestras de material verde, que fueron depositadas en el Herbario de la Universidad de Brasilia. Paralelamente se inició una colecta sistematizada de muestras de maderas de las especies que ingresaban por primera vez en las listas correspondientes del herbario. Para proceder a la recogida de las muestras de madera se extrajo del fuste de cada árbol un disco o rodaja, a la altura de 1,30 $\mathrm{m}$ y de $5 \mathrm{~cm}$ de espesor aproximadamente, que comprendía la sección transversal completa del tronco. Fue apeado un árbol adulto por cada una de las especies objeto de estudio. Los árboles procedían de las regiones norte, centro y nordeste del Brasil.

Los discos fueron depositados en la xiloteca del Laboratorio de Anatomía de la Madera de la Universidad de Brasilia y posteriormente fueron transportados a carpintería, donde se cortó de cada uno de ellos una probeta de ensayo en forma de cuña que abarcaba desde la médula hasta la corteza (Fig. 1). Para extraer la probeta de ensayo se escogió en el disco una zona donde la presencia de singularidades de la madera fuera mínima.

De cada probeta con forma de cuña se extrajeron tres cilindros (Fig. 1), de dimensiones dependientes del tamaño del disco. Los cilindros fueron secados en estufa a $105^{\circ} \mathrm{C}$ $\pm 2{ }^{\circ} \mathrm{C}$ hasta llegar al estado anhidro (Associaçao Brasileira de Normas Técnicas [ABNT], 2003); posteriormente

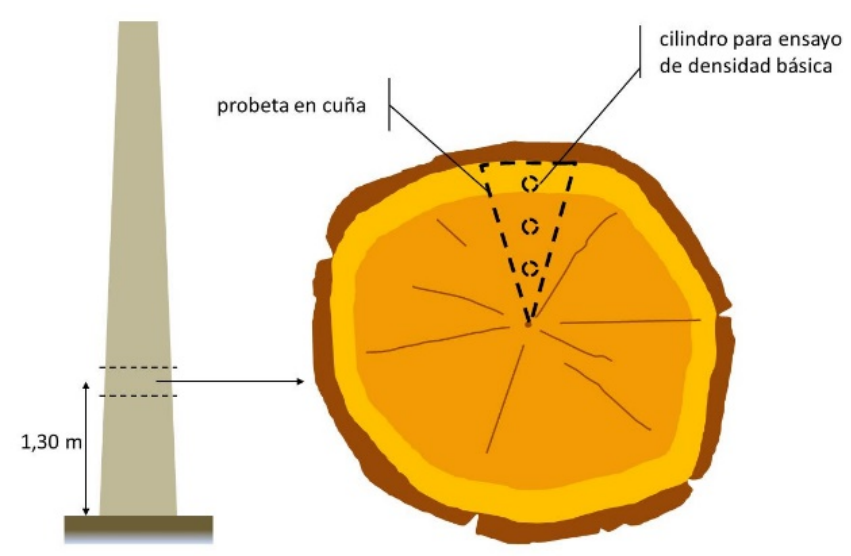

FIGURA 1. Esquema de la zona de extracción del disco en el fuste y de la sección transversal del disco, con la localización de la cuña y de las probetas de ensayo cilíndricas a extraer para efectuar la determinación de la densidad básica. 
fueron pesados en balanza con resolución de $1 \mathrm{mg}$ para obtener la masa anhidra. A continuación, fueron sumergidos en agua durante 48 horas. Pasado el tiempo de inmersión se obtuvieron las dimensiones de los cilindros, calculando su volumen por el principio de Arquímedes. La densidad básica fue calculada como cociente entre la masa anhidra y el volumen saturado de humedad (ABNT, 2003), como la densidad básica es relativa a la densidad del agua, no tiene unidades (Panshin y DeZeew, 1970). Tal como establece la norma de ensayo referida, la madera analizada no fue sometida a un proceso previo de extracción de las sustancias de impregnación y otros componentes minoritarios ya que al extraerlos se estaría analizando un material diferente de la madera en su estado natural (Williamson y Wiemann, 2010). Durante la fase de secado en estufa a más de $100{ }^{\circ} \mathrm{C}$ se espera la volatilización de algunas sustancias constituyentes de la madera que tienen bajo peso molecular. A cada especie se le asignó como densidad básica la media aritmética de los valores de densidad básica obtenidos sobre los tres cilindros.

El proceso de identificación de la especie botánica a la que pertenecía cada muestra de madera fue especialmente meticuloso y se basó en la identificación en campo por un dendrólogo - taxónomo (uno de los autores del presente trabajo), así como en el reconocimiento de las muestras herborizadas. La experiencia de los autores refleja que en los inventarios forestales los errores en la identificación de especies son muy frecuentes, ya sea por la dificultad inherente a la identificación de taxones en bosques pluriespecíficos o bien debido a que los inventariadores de recursos madereros basan su identificación solamente en el testimonio de la población local, que suele asignar el mismo nombre a un grupo más o menos amplio de especies botánicas. Esta imprecisión en las denominaciones lleva a una clasificación errónea de las especies, lo cual puede ser accidental o intencionado, ya que bajo un mismo nombre popular pueden extraerse en un mismo lote especies de maderas cuyo aprovechamiento puede estar hasta prohibido, como es el caso del mogno o caoba (Swietenia macrophylla) o maderas de inferior calidad a la que correspondería por el nombre comercial. Un ejemplo de agrupación bajo un mismo nombre comercial en Brasil es la denominación amescla para las especies de los géneros Protium y Trattinnickia (Hornink, Santini Jr. y Tomazello Filho, 2015).

\section{Resultados}

Sesenta y ocho por ciento de las especies analizadas (40 de 59 especies) presentan maderas livianas o semipesadas (densidad básica entre 0,51 y 0,80) (Tabla 1; Fig. 2).

TABLA 1. Distribución de las 59 especies arbóreas analizadas de seis familias del Orden Sapindales en función de la densidad básica de la madera.

\begin{tabular}{|c|c|c|c|c|c|c|c|}
\hline \multirow{2}{*}{$\begin{array}{l}\text { clase de } \\
\text { densidad }^{(l)}\end{array}$} & \multirow[t]{2}{*}{ densidad básica } & \multicolumn{6}{|c|}{ número de especies } \\
\hline & & 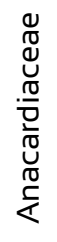 & 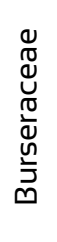 & 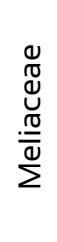 & 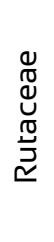 & 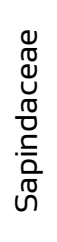 & 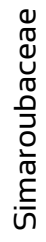 \\
\hline muy liviana & $0,30-0,50$ & 2 & $\mathrm{O}$ & 3 & 0 & 0 & 1 \\
\hline liviana & $0,51-0,65$ & 4 & 8 & 3 & 2 & $\mathrm{O}$ & 2 \\
\hline semipesada & $0,66-0,80$ & 5 & 5 & 4 & 3 & 4 & $\mathrm{O}$ \\
\hline pesada & $0,81-0,95$ & 5 & 0 & 0 & 2 & 1 & 0 \\
\hline muy pesada & $>0,95$ & 2 & 1 & 0 & 2 & 0 & $\mathrm{O}$ \\
\hline Total & & 18 & 14 & 10 & 9 & 5 & 3 \\
\hline
\end{tabular}




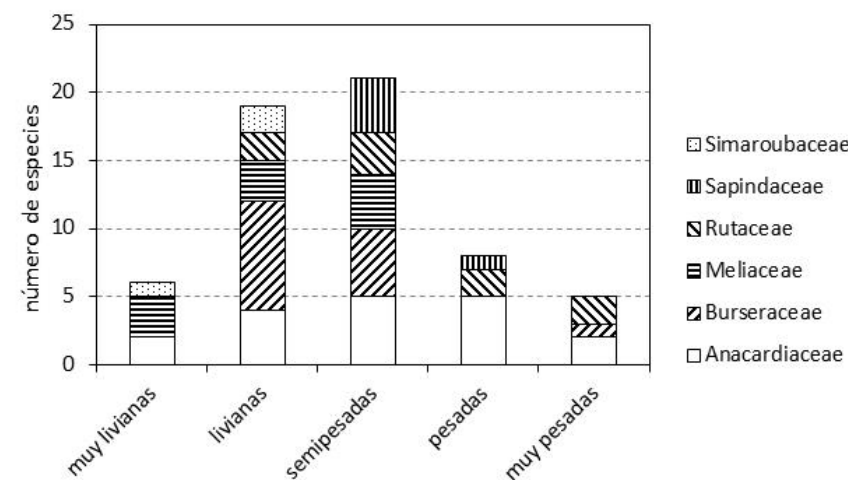

FIGURA 2. Distribución de las especies por clases de densidad básica y por familias.

En el total de las seis familias botánicas analizadas la madera presenta en promedio una densidad de 0,71 , siendo en su conjunto más ligeras las maderas de la familia Simaroubaceae $(0,51)$, seguidas de Meliaceae $(0,60)$, Burseraceae $(0,71)$, Anacardiaceae $(0,75)$, Rutaceae $(0,79)$ y Sapindaceae $(0,80)$. En la figura 3 se muestra la abundancia relativa en cada familia de las diferentes clases de maderas por su densidad, atendiendo al criterio de clasificación dado por la norma NBR 11941 (ABNT, 2003). El intervalo de variación de densidades se encuentra entre los 0,38 de la madera de Simarouba amara y los 1,25 de Schinopsis lorentzii (Tabla 2, Fig. 4; Fig. 5). Si la densidad es diferente entre especies, es muy probable que también sean diferentes las demás propiedades de la madera, dada la relación que estas presentan con la densidad (Desch y Dinwoodie, 1996; Forest Products Laboratory, 2010; Zobel y Buijtenen, 1989).

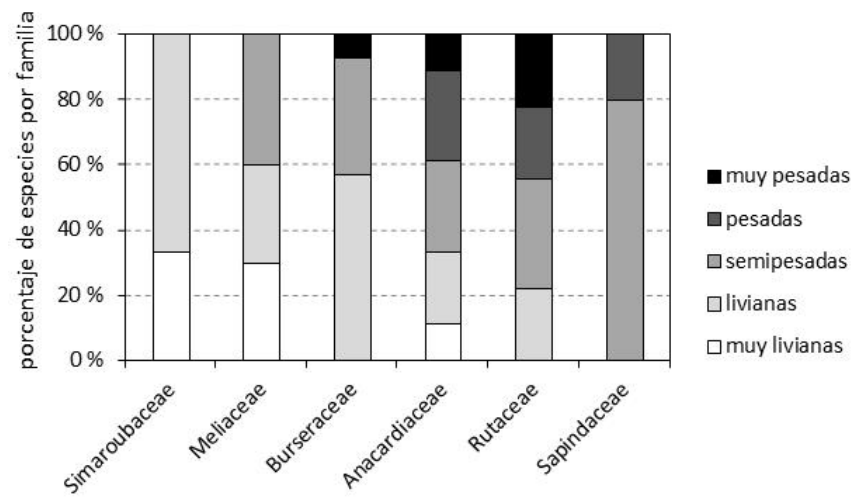

FIGURA 3. Abundancia relativa de las diferentes especies en cada familia por clase de densidad básica.
Las columnas en blanco representan la media de los resultados experimentales y las columnas negras representan el intervalo de valores recopilados en bibliografía. Las familias y cada especie en cada familia están ordenadas por densidad básica creciente.

Las columnas en blanco representan la media de los resultados experimentales y las columnas negras representan el rango de valores recopilados en bibliografía. Las familias y cada especie en cada familia están ordenadas por densidad básica creciente.

El intervalo de variación de densidades en el material analizado es claramente superior en la familia Anacardiaceae, que presenta maderas pertenecientes a todas las clases de densidad y es también la familia más representada en la muestra (Fig. 5). Por el contrario, las familias que presentan más homogeneidad en cuanto a densidad básica de la madera son Simaroubaceae y Sapindaceae, que también son las familias menos representadas en la muestra (Fig. 4; Fig. 5). De hecho, se ha encontrado una estrecha relación entre intervalo de variación de densidad entre especies de una familia y número de especies analizadas, lo que indica la necesidad de tener en cuenta el número de especies evaluadas en los estudios de variación interespecífica de las propiedades de la madera.

\section{DISCUSIÓN}

No es muy abundante la bibliografía en torno a propiedades físicas de la madera de los taxones objeto de estudio. De hecho, no se han encontrado referencias sobre densidad básica para 15 de las 59 especies analizadas y solo en 30 de las especies de la muestra se localizó más de una referencia (Tabla 2, Fig. 4; Fig. 5). Por tanto, el presente trabajo constituye una significativa aportación al conocimiento de la madera de ciertas especies forestales de Brasil que han sido escasamente estudiadas.

Los valores experimentales de densidad básica son en promedio $21 \%$ superiores a los valores recogidos en las referencias bibliográficas, aunque existe una variación que va desde el extremo inferior, que es el caso de la madera de Astronium graveolens, para la que se ha encontrado que la 


Madera y Bosques vol. 25, núm. 2, e2521817 Verano 2019

TABLA 2. Densidad básica de la madera de 59 especies arbóreas pertenecientes a seis familias del Orden Sapindales. Los nombres científicos son conformes con la nomenclatura botánica del Jardín Botánico de Missouri (Estados Unidos).

\begin{tabular}{|c|c|c|}
\hline \multirow[t]{2}{*}{ Taxones } & \multicolumn{2}{|c|}{ densidad básica } \\
\hline & $d b$ & bibliografía \\
\hline \multicolumn{3}{|l|}{ Familia Anacardiaceae } \\
\hline Anacardium curatellifolium St. Hil & 0,64 & \\
\hline Anacardium excelsum Standl. & 0,56 & $0,32-0,48^{(1)} ; 0,34^{(2)(3)} ; 0,41^{(4)(5)} ; 0,44-0,55^{(6)}$ \\
\hline Anacardium giganteum Haancock. & 0,56 & $0,38-0,52^{(1)} ; 0,41^{(7)} ; 0,44^{(4)} ; 0,48^{(5)}$ \\
\hline Anacardium macrocarpum Ducke & 0,68 & \\
\hline Anacardium negrense Pires \& Frós & 0,41 & \\
\hline Anacardium occidentale L. & 0,67 & $0,41-0,50^{(1)}$ \\
\hline Astronium fraxinifolium Schott. & 0,95 & $0,91^{(15)}$ \\
\hline Astronium graveolens Jacq. & 0,73 & $0,73-1,09^{(1)} ; 0,75^{(4)} ; 0,75-0,89^{(7)} ; 0,84^{(2)} ; 0,86^{(5)}$ \\
\hline Astronium lecointei Ducke & 0,83 & $0,73^{(4)(7)} ; 0,73-0,98^{(1)} ; 0,81^{(5)}$ \\
\hline Astronium urundeuva (Allemao) Engl. & 1,16 & $0,69-1,19^{(1)} ; 0,75-0,95^{(6)} ; 1,00^{(5)} ; 1,21^{(4)}$ \\
\hline Fusea longifolia (Aublet) Saff & 0,82 & $0,74^{(1)}$ \\
\hline Lithrea molleoides (Vell.) Engler & 0,81 & $0,41-0,98^{(1)}$ \\
\hline Mangifera indica $\mathrm{L}$. & 0,68 & $0,41-0,98^{(1)} ; 0,52-0,68^{(1)} ; 0,55^{(4)(7)}$ \\
\hline Schinopsis lorentzii Engler & 1,25 & $0,90^{(1)}$ \\
\hline Spondias lutea Linn. & 0,42 & $0,38^{(4)(7)} ; 0,40^{(5)(6)}$ \\
\hline Tapirira guianensis Aubl. & 0,51 & $0,40^{(3)} ; 0,41-0,55^{(1)} ; 0,46^{(5)} ; 0,47^{(7)} ; 0,50^{(4)} ; 0,55-0,75^{(6)}$ \\
\hline Tapirira obtusa (Benth.) Mitch. & 0,94 & $0,29^{(1)}$ \\
\hline Thyrsodium schomburgkianum Benth. & 0,79 & \\
\hline \multicolumn{3}{|l|}{ Familia Burseraceae } \\
\hline Bursera tecomaca Standl. & 0,78 & \\
\hline $\begin{array}{l}\text { Hemicrepidospermum rhoifolium (Benth.) } \\
\text { Swartze } \\
\text { Protium araguense Cartt. }\end{array}$ & $\begin{array}{l}0,77 \\
0,65\end{array}$ & \\
\hline Protium brasiliense (Spreng.) Engler & 0,65 & $0,72-0,77^{(1)}$ \\
\hline Protium hebetatum D. Daly & 0,64 & \\
\hline Protium heptaphyllum (Aublet) Mach. & 0,63 & $0,40-0,55^{(7)} ; 0,47-0,87^{(1)} ; 0,54^{(4)}$ \\
\hline Protium insigne Engler & 0,63 & \\
\hline Protium paniculatum Engl. & 0,65 & $0,49^{(1)}$ \\
\hline Protium paraense Cuatrec. & 0,65 & $0,51^{(1)}$ \\
\hline Protium puncticulatum March. & 0,56 & \\
\hline Tetragastris altissima (Aublet) Swartz. & 0,75 & $0,61^{(7)} ; 0,61-0,75^{(1)} ; 0,74^{(4)(8)}$ \\
\hline Tetragastris panamense O. Kunt. & 0,77 & $0,43-0,47^{(1)} ; 0,61^{(7)} ; 0,61-0,75^{(1)} ; 0,70^{(9)} ; 0,74^{(4)}$ \\
\hline Tetragastris paraensis Engler & 1,03 & $0,92^{(16)}$ \\
\hline Trattinikia burseraefolia (Mart.) Willd. & 0,78 & $0,43-0,50^{(1)} ; 0,44^{(7)(10)} ; 0,50^{(5)}$ \\
\hline \multicolumn{3}{|l|}{ Familia Meliaceae } \\
\hline Cabralea canjerana Saldanha & 0,67 & $0,41-0,69^{(1)} ; 0,55^{(4)(7)} ; 0,55-0,75^{(6)} ; 0,61^{(5)}$ \\
\hline Carapa guianensis Aublet & 0,63 & $0,43^{(10)} ; 0,49^{(3)(5)(9)(13) ;} ; 0,53^{(1)} ; 0,55^{(4)} ; 0,56^{(7)}$ \\
\hline Cedrela fissilis Vell. & 0,48 & $0,42-0,55^{(1)}$ \\
\hline Cedrela huberi Ducke & 0,42 & \\
\hline
\end{tabular}




$\begin{array}{lll}\text { Cedrela odorata L. } & 0,66 & 0,34-0,66^{(1)} ; 0,38^{(3)(10)} ; 0,39^{(8)} ; 0,40-0,75^{(6)} ; 0,42^{(4)(5)} ; 0,43-0,45^{(7)} \\ \text { Cedrela tonduziii DC. } & 0,48 & 0,36^{(1)(14)} \\ \text { Guarea trichilioides L. } & 0,72 & 0,36-0,56^{(1)} ; 0,40-0,55^{(6)} ; 0,48^{(5)} ; 0,51-0,52^{(7)} ; 0,53^{(3)} ; 0,66^{(8)(10)} \\ \text { Melia azedarach L. } & 0,59 & 0,48^{(17)} \\ \text { Swietenia macrophylla King } & 0,63 & 0,42-54^{(7)} ; 0,43^{(4)} ; 0,43-0,63^{(1)} ; 0,52^{(5)(8)} \\ \text { Trichilia catigua A. Juss. } & 0,71 & 0,69^{(1)}\end{array}$

Familia Rutaceae

\begin{tabular}{lll}
\hline Balfourodendron riedelianum Engler & 0,88 & $0,68^{(1)} ; 0,74^{(5)}$ \\
Esenbeckia leiocarpa Engler & 1,09 & $0,96^{(1)}$ \\
Euxylophora paraensis Huber & 0,72 & $0,52-0,70^{(1)} ; 0,69^{(5)}$ \\
Faramea cyanea Müll. Arg. & 0,96 & \\
Metrodorea flavida K. Krause & 0,80 & \\
Spathelia excelsa (Krause) R. S. Cowan \& Briz. & 0,88 & \\
Zanthoxylum pentandrum (Aubl.) R.A. Howard & 0,66 & $0,65^{(1)}$ \\
Zanthoxylum regnellianum Engler & 0,53 & $0,50^{(1)}$ \\
Zanthoxylon rhoifolium Lam. & 0,58 & $0,56^{(1)}$
\end{tabular}

Familia Sapindaceae

Cupania vernalis (Camb.) St. Hil.

Diplokeleba floribunda N. E. Brow

Matayba arborescens (Aublet) Radlk

Sapindus saponaria $\mathrm{L}$.

Talisia esculenta Radl.

$\begin{array}{ll}0,72 & 0,65-0,67^{(1)} \\ 0,91 & 0,83-0,87^{(1)} \\ 0,78 & 0,60-0,76^{(1)} \\ 0,78 & 0,58^{(4)} ; 0,60-0,83^{(1)} \\ 0,79 & 0,67-0,95^{(1)}\end{array}$

$0,72 \quad 0,65-0,67^{(1)}$

$0,780.000,76$

$0,78 \quad 0,58^{(4)} ; 0,60-0,83^{(1)}$

$0,67-0,95$
Familia Simaroubaceae

Picramia junimiana Macbr.

Simarouba amara Aubl. Simarouba cedron PI.

$\begin{array}{ll}0,56 & \\ 0,38 & 0,32-0,38^{(7)} ; 0,35^{(9)(10)} ; 0,35-0,50^{(1)} ; 0,36^{(4)(9)(11)} ; 0,39^{(5)} ; 0,42-0,58^{(12)} \\ 0,58 & 0,47^{(1)}\end{array}$

$0,58 \quad 0,47^{(1)}$

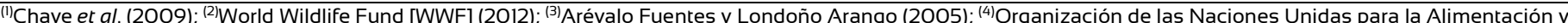

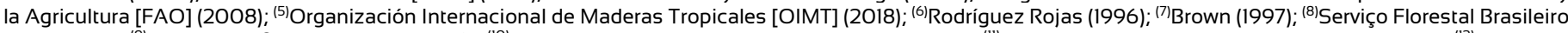

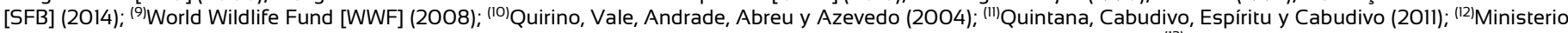

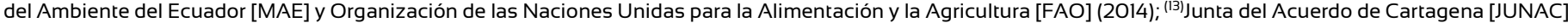
(1981); ${ }^{(14)}$ Carpio (2003); ${ }^{(15)}$ Baeta y Santos (1999); ${ }^{(16)}$ Carvalho (2004); ${ }^{(17)}$ Atencia (2003). db: promedio de los resultados experimentales.

densidad básica es 14\% más baja que la media de las siete referencias bibliográficas localizadas para la especie. Por otro lado se encuentra Tapirira obtusa, cuya densidad básica en la muestra triplica el valor recogido en la única referencia bibliográfica disponible (Fig. 5). Las mayores discrepancias entre resultados experimentales y bibliografía se encuentran en la familia Anacardiaceae, la más numerosa de la muestra, en la que la diferencia de densidades llevaría a clasificar a las respectivas maderas en clases de densidad diferentes. Así mismo, las menores discrepancias entre datos experimentales y bibliografía se encuentran en una de las familias menos representadas (Sapindaceae) (Tabla 2 y Fig. 5). Esta relación positiva entre número de especies analizadas por familia y desviación de resultado experimental respecto de resultado bibliográfico es explicable dado que al aumentar el número de especies analizadas crece la probabilidad de incluir en el análisis valores (experimentales o bibliográficos) alejados de los promedios. Con todo, la mayor o menor representatividad de la muestra en cada familia no es suficiente para explicar 


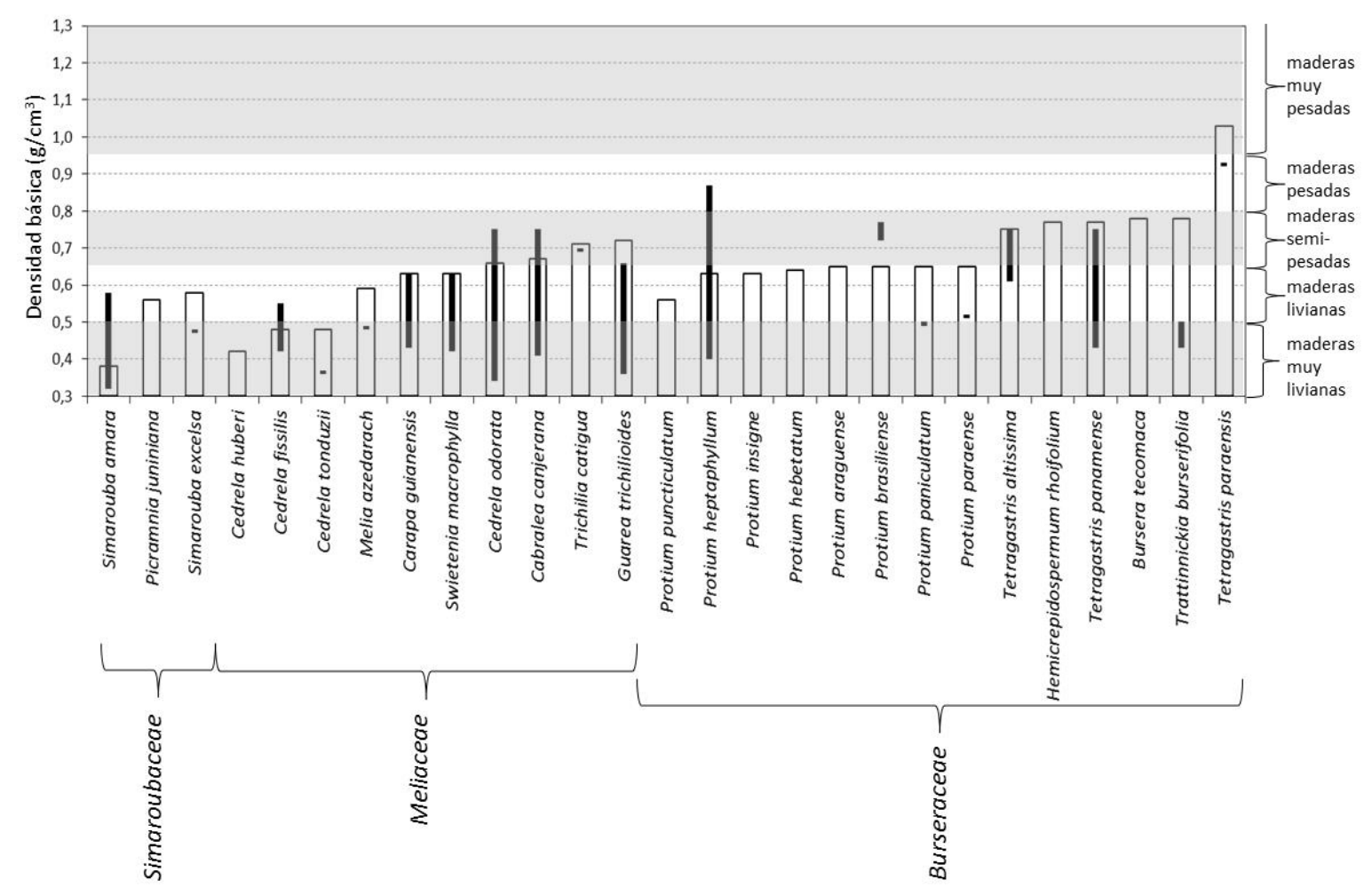

FIGURA 4. Densidad básica de la madera de las especies analizadas para las tres familias con maderas más livianas (Simaroubaceae, Meliaceae y Burseraceae).

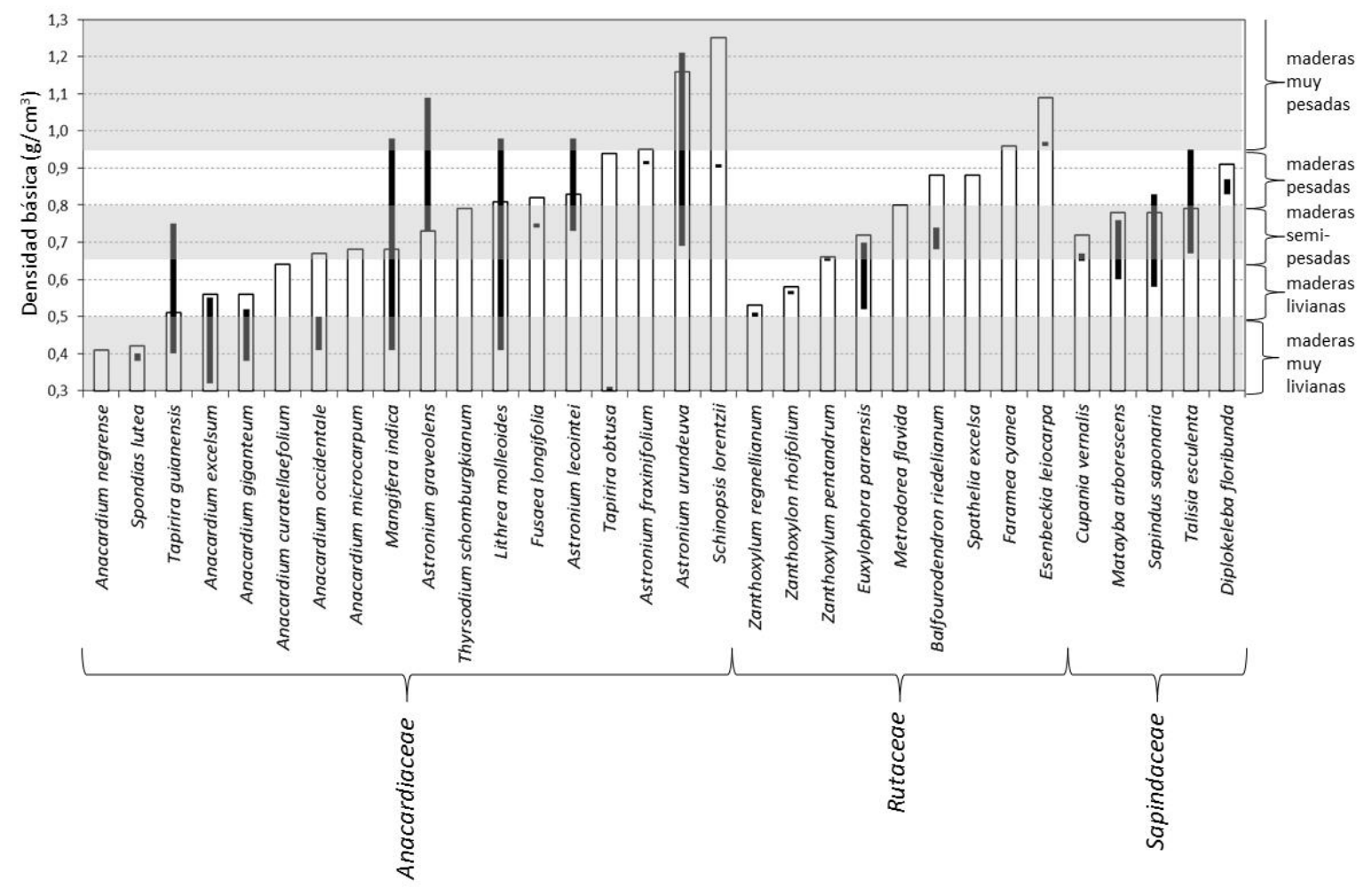

FIGURA 5. Densidad básica de la madera de las especies analizadas para las tres familias con maderas más pesadas (Anacardiaceae, Rutaceae y Sapindaceae). 
las discrepancias halladas entre resultados experimentales y bibliografía ya que se ha analizado una parte importante de los géneros y especies del orden Sapindales presentes en Brasil (Tomazello Filho et al., 2000). Por tanto, las diferencias positivas de densidad básica entre la madera analizada y las referencias de bibliografía han de atribuirse en parte a que la muestra de este estudio procede de una posición en el tronco (1,30 $\mathrm{m}$ de altura) donde la densidad de la madera es superior a la media del fuste completo, dado que la edad cambial es mayor en la base del árbol y la densidad está muy relacionada con la edad cambial, como se indicó en la Introducción (Desch y Dinwoodie, 1996; Kollmann, 1959). Así mismo, parte de las discrepancias de densidad básica pueden atribuirse a diferencias de edad entre los árboles analizados en bibliografía y los árboles analizados en este estudio (cuya edad se desconoce), dado que la edad del árbol constituye una importante fuente de variación de la densidad de la madera (Tomazello Filho et al., 2000). Finalmente, los valores de densidad obtenidos en este trabajo corresponden a madera en la que no se han eliminado los extractivos, como se ha indicado en Materiales y Métodos, con lo cual se presentan valores superiores a los que se obtendrían con madera extraída. Sin embargo, no se cree que el factor presencia o ausencia de extractivos en la madera sea la principal explicación de las discrepancias halladas en cuanto a densidad de la madera entre datos experimentales y bibliográficos ya que el peso de los extractivos en la madera suele ser inferior a $2 \%$ (Williamson y Wiemann, 2010) por lo que las sustancias de extracción explicarían solo una pequeña parte de las discrepancias entre datos experimentales y bibliográficos.

Por lo anterior, se hace necesario el estudio de las procedencias brasileñas de las especies de maderas de estas familias, especialmente de Anacardiaceae, ya que la información procedente de bibliografía no siempre describe de forma precisa la madera de origen brasileño de las especies botánicas consideradas.

\section{CONCLUSIONES}

Sesenta y ocho por ciento de las especies forestales analizadas presentan maderas livianas y semipesadas, siendo la familia Simaroubaceae la que en promedio presenta las maderas más livianas y las familias Rutaceae y Sapindaceae las que en promedio presentan las maderas más pesadas y las que más eficazmente contribuyen, por tanto, a la fijación del carbono atmosférico en forma de biomasa. En la familia Anacardiaceae, la más numerosa en la muestra, se dieron las mayores diferencias de densidad básica entre especies. Tanto la variabilidad interespecífica de la densidad básica como las desviaciones respecto de los resultados bibliográficos crecen cuanto mayor es el número de especies analizadas, lo que apunta a la necesidad de abarcar un amplio número de especies en los estudios sobre densidad de la madera procedente de bosques pluriespecíficos. Los valores de densidad obtenidos constituyen una significativa aportación al conocimiento de este conjunto de maderas tropicales ya que para una cuarta parte de las especies analizadas no se encontraron referencias bibliográficas sobre densidad básica de la madera. En futuros inventarios forestales dirigidos a conocer el volumen de existencias maderables será posible emplear los valores de densidad básica aquí obtenidos para estimar las toneladas de materia leñosa correspondientes, bajo el supuesto de una correcta identificación de las especies botánicas inventariadas. En los nuevos estudios sobre densidad de la madera de especies de procedencia brasileña será conveniente estimar la edad de los individuos objeto de análisis y tomar muestras a diferentes alturas en el fuste para efectuar comparaciones con maderas de otras procedencias, considerando así mismo que la presencia de extractivos pudiera afectar significativamente a la densidad en algunas familias.

\section{REFERENCIAS}

Arévalo Fuentes, R. L., \& Londoño Arango, A. (2005). Manual para la identificación de maderas que se comercializan en el Departamento del Tolima. Ibagué, Colombia: Universidad del Tolima.

Associaçao Brasileira de Normas Técnicas [ABNT]. (2003). NBR 11941: madeira, determinaçao da densidade básica. Rio de Janeiro: ABNT.

Atencia, M. E. (2003). Densidad de maderas $\left(\mathrm{kg} / \mathrm{m}^{3}\right)$ ordenadas por nombre común. Buenos Aires, Argentina: INTI/CITEMA. 
Baeta, I. da C., \& Santos, V. (1999). Resistencia dos materiais e dimensionamento de estruturas para construcoes rurais. Viçosa, Brasil: Universidade Federal de Viçosa, Depto. Engenharia Agrícola.

Brown, S. (1997). Estimating biomass and biomass change of tropical forest: a primer. FAO Forestry Paper 134. Roma, Italia: FAO.

Carpio, M. I. M. (2003). Maderas de Costa Rica: 150 especies forestales ( $2^{\mathrm{a}}$ ed.). San José de Costa Rica, Costa Rica: Editorial Universidad de Costa Rica.

Carvalho, G. (2004). Tabela de peso especifico de alumas madeiras da Amazonia. Belém, Brasil: AIMEX.

Chave, J., Coomes, D. A., Jansen, S., Lewis, S. L., Swenson, N. G., \& Zanne, A.E. (2009). Towards a wordwide wood economics spectrum (Global Wood Density Database). Ecology Letters, 12(4), 351-366. doi:10.1111/j.14610248.2009.01285.x

Desch, H. E., \& Dinwoodie, J. M. (1996). Timber. Structure, properties, conversion and use. Hong-Kong, China: MacMillan Press Ltd.

Devesa Alcaraz, J. A., \& Carrión García, J. S. (2012). Las plantas con flor: apuntes sobre su origen, clasificación y diversidad. Córdoba, España: Servicio de Publicaciones de la Universidad de Córdoba.

Eckardt, M., Rodrigues Nascimento, H., Nazário de Alameida, A., \& Vieira de Abreu, Y. (2015). Crescimento da actividade florestal no Brasil. Revista da madeira, 145, 20-23.

Forest Products Laboratory. (2010). Wood handbook. Wood as an engineering material. USD A For. Serv. Gen. Tech. Rep. FPL-GTR113. Madison, Estados Unidos: USDA.

Harrington, M. (2008). Phylogeny and evolutionary history of Sapindaceae and Dodonaea. Tesis Doctoral, James Cook University, Townsville, Australia.

Hornink, B., Santini Jr., L., \& Tomazello Filho, M. (2015). Análise da estrutura anatômica das madeiras comerciais no Estado de São Paulo e suas aplicações. En $23^{\circ}$ Simpósio Internacional de Iniciação Científica da USP. Piracicaba: SIICUSP.

Izco, J. (2009). Botánica (2o ed.). Madrid, España: McGraw-Hill Interamericana.

Junta del Acuerdo de Cartagena [Junac]. (1981). Tablas de propiedades físicas y mecánicas de la madera de 24 especies de Colombia. Lima: Junac.

Kollmann, F. (1959). Tecnología de la madera y sus aplicaciones. Madrid, España: Ministerio de Agricultura.

Li, S., Lens, F., Espino, S., Karimi, Z., Klepsch, M., Jochen Schenk, H., Schmitt, M., Schuldt, B., \& Jansen, S. (2016).
Intervessel pit membrane thickness as a key determinant of embolism resistance in angiosperm xylem. IAW A Journal, 37(2), 152-171. doi: 10.1163/22941932-20160128

Martínez-Cabrera, H. I., Jones, C. S., Espino, S., \& Jochen Schenk, J. (2009). Wood anatomy and wood density in shrubs: responses to varying aridity along transcontinental transects. American Journal of Botany, 96, 1388-1398. doi:10.3732/ajb.0800237

Mettem, C. J., \& Richens, A. D. (1991). Hardwoods in construction. High Wycombe, Reino Unido: Timber Research \& Development Association.

Miller, N. G. (1990). The genera of Meliaceae in the southeastern United States. Journal of the Arnold Arboretum, 52, 453-486.

Ministerio del Ambiente del Ecuador [MAE], Organización de las Naciones Unidas para la Alimentación y la Agricultura [FAO]. (2014). Propiedades anatómicas, físicas y mecánicas de 93 especies forestales. Ecuador: MAE-FAO.

Organización de las Naciones Unidas para la Alimentación y la Agricultura [FAO]. (2008). Evaluación de los recursos forestales nacionales 2010. Directrices para la elaboración de informes nacionales destinados a FRA 2010. Roma: FAO, Departamento de Bosques.

Organización Internacional de Maderas Tropicales [OIMT]. (2018). Lesser used species. Recuperado de http://www.tropicaltimber.info/es/

Panshin, A. J., \& de Zeeuw, C. (1970). Textbook of Wood technology. Volume 1. (3a ed.). Nueva York: McGraw-Hill Book Company

Peraza Oramas, C., \& González Álvarez, M. A. (1973). Tecnología de la madera. Volumen I. La producción maderera y su importancia económica. Madrid, España: AITIM.

Quintana, S., Cabudivo, A., Espíritu, J. M., \& Cabudivo, J. M. (2011). Propiedades físico- mecánicas de las maderas de Simarouba amara (Aubl.) y Cedrelinga catenaeformis (Ducke) de plantaciones de diferentes edades, San Juan Bautista, Loreto, Perú. Conocimiento Amazónico, 2(2), 115-123.

Quirino, W. F., Vale, A. T., Andrade, A. P. A., Abreu, V. L. S., \& Azevedo, M. C. S. (2004). Poder calorífico da madeira e de resíduos lignocelolósicos. Biomassa e Energia, 1(2), 173-182.

Rech, C. (2015). Manejo adequado pode reducir desmatamento na Amazônia. Revista da madeira, 145, 42.

Rodríguez Rojas, M. (1996). Manual de identificación de especies forestales de la subregión andina. Lima, Perú: Instituto Nacional de Investigación Agraria, Organización Internacional de las Maderas Tropicales. 
Savi, T., Love, V. L., Dal Borgo, A., Martellos, S., \& Nardini, A. (2017). Morpho-anatomical and physiological traits in saplings of drought-tolerant Mediterranean woody species. Trees, 31, 1137-1148. doi:10.1007/s00468-017-1533-7

Serviço Florestal Brasileiro [SFB]. (2014). Madeiras tropicales brasileiras, v.2. Brasilia, Brasil: SFB/LPF.

Strasburger, E., \& Sitte, P. (2004). Tratado de botánica (9a ed.). Barcelona, España: Omega.

Tomazello Filho, M., Botosso, P. C., \& Lisi, C. S. (2000). Potencialidade da família Meliaceae para Dendrocronologia em regiões tropicais e subtropicais. En F. A. Roig (Ed.), Dendrocronología en America Latina (pp. 381-431). Mendoza: Ediunc.

Williamson, G. B., \& Wiemann, M. C. (2010). Measuring wood specific gravity ... correctly. American Journal of Botany, 97(3): 519-524. doi:10.3732/ajb.0900243

World Wildlife Fund [WWF] (2008). Maderas de Colombia. Global Forest and Trade Network. Bogotá. WWF.
World Wildlife Fund [WWF] (2012). Maderas de Panamá. Global Forest and Trade Network. Ciudad de Panamá: WWF.

Zobel, B. J., \& Buijtenen, J. P. V. (1989). Wood variation. Its causes and control. Berlín, Alemania: Springer Verlag.

Zomlefer, W. B. (2004). Guía de las familias de plantas con flor. Zaragoza, España: Acribia.

Manuscrito recibido el 6 de julio de 2018

Aceptado el 22 de septiembre de 2018

Publicado el 29 de julio de 2019

Este documento se debe citar como:

Riesco M., G., Imaña E., J., \& de Paula, J. E. (2019). Densidad de la madera de 59 especies del orden Sapindales procedentes de bosques naturales brasileños. Madera y Bosques, 25(2), e2521817. doi: $10.21829 /$ myb.2019.2521817

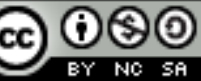

Madera y Bosques por Instituto de Ecología, A.C. se distribuye bajo una Licencia Creative Commons Atribución-NoComercialCompartirlgual 4.0 Internacional. 